Pacific Journal of Mathematics

SPLINES AND THE LOGARITHMIC FUNCTION

November 1975 


\section{SPLINES AND THE LOGARITHMIC FUNCTION}

\section{J. Newman and I. J. Schoenberg}

The paper studies a spline function $S_{n}(x)(0<x<\infty)$ of degree $n$, with knots at the points of the geometric progression $x_{k}=q^{k}(q$ is fixed $>1, k=0, \pm 1, \pm 2, \cdots)$, which in shown to be uniquely defined by the following two properties: $1^{\circ}$. $S_{n}(x)$ interpolates the function $f(x)=\log x / \log q$ at all the knots $q^{k}, 2^{\circ}$. $S_{n}(x)$ satisfies the functional equation $S_{n}(q x)=$ $S_{n}(x)+1$ for $x>0 . \quad S_{n}(x)$ is explicitly determined and shown to share with $f(x)$ some of its global properties. The main point is the detailed study of the somewhat surprising behavior of $S_{n}(x)$ as $n \rightarrow \infty$.

Introduction. Spline interpolants of exponential functions were discussed in [3], Lecture 3, and were found to be useful in a study of cardinal spline interpolation ([3], Lecture 4). Here we attempt to interpolate by splines the logarithmic function $\log x(0<x<\infty)$. As the domain of definition of $\log x$ is the positive halfline we do not use the usual cardinal polynomial splines, but rather so-called cardinal $q$-splines defined as follows.

Let $n$ be a natural number and $q$ be real, $q>1$. We denote by

$$
\sum_{n, q}=\{S(x)\}
$$

the class of functions $S(x)$, defined on the half-axis $0<x<\infty$, and satisfying the following two conditions:

1. The restriction of $S(x)$ to every interval $\left(q^{\nu}, q^{\nu+1}\right)$ ( $\nu$ any integer) is a polynomial of degree at most $n$.

2.

$$
S(x) \in C^{n-1}(0, \infty)
$$

Such functions $S(x)$ are commonly called spline functions (or splines) of degree $n$ having as knots the points $q^{\nu}(-\infty<\nu<\infty)$. We also call them $q$-splines to remind us of the nature of their knots. Finally, the term cardinal $q$-splines is to indicate that the domain of $S(x)$ is $(0, \infty)$ and that all knots $q^{\nu}$ many occur. We now propose the following

Problem 1. To find

$$
S_{n}(x) \in \sum_{n, q}
$$

such as to interpolate the function 


$$
f(x)=\frac{\log x}{\log q}
$$

at the points $\left(q^{\nu}\right)$, hence such that

$$
S_{n}\left(q^{\nu}\right)=\nu \text { for all integers } \nu .
$$

As stated this problem admits infinitely many solutions. Indeed, a moment's reflexion (see [3], Lemma 1.1 on p. 33) will show that we obtain all solutions of Problem 1 as follows. The symbol $\pi_{n}$ denoting the class of polynomials of degree $\leqq n$, let $P(x)$ be an arbitrary element of $\pi_{n}$ such that

$$
P(1)=0, \quad P(q)=1,
$$

and let

$$
\begin{aligned}
S(x)= & P(x)+a_{1}(x-q)_{+}^{n}+a_{2}\left(x-q^{2}\right)_{+}^{n}+\cdots \\
& +a_{0}(1-x)_{+}^{n}+a_{-1}\left(q^{-1}-x\right)_{+}^{n}+\cdots, \quad(0<x<\infty),
\end{aligned}
$$

where we use the notation $u_{+}=\max (u, 0)$. In the interval $[1, q]$ all truncated powers vanish and so $S(x)=P(x)$. In the interval $\left[q, q^{2}\right]$, besides $P(x)$, only the term $a_{1}(x-q)_{+}^{n}$ does not vanish and we can determine $a_{1}$ uniquely by requiring that $S\left(q^{2}\right)=2$. Next we determin $a_{2}$ uniquely from $S\left(q^{3}\right)=3$, a.s.f. Likewise the coefficients $a_{0}, a_{-1}, \cdots$ are successively and uniquely determined by the interpolatory condition $S\left(q^{-1}\right)=-1, S\left(q^{-2}\right)=-2, \ldots$. We see that the interpolating $q$-spline $S(x)$ is uniquely defined once we have chosen $P(x) \in \pi_{n}$ such as to satisfy (6).

Clearly Problem 1 will become meaningful only if we impose on $S_{n}(x)$ further conditions. This we do as follows. We observe that the function (4) satisfies the functional equation $f(q x)=f(x)+1$. This suggests that we require the $q$-spline $S_{n}(x)$ to satisfy the functional equation

$$
S_{n}(q x)=S_{n}(x)+1, \quad(0<x<\infty) .
$$

It should be noticed that if (8) is satisfied and also

$$
S_{n}(1)=0,
$$

then all relations (5) evidently follow.

The contents of the seven sections of this paper are briefly as follows. In $\S 1$ we construct the unique $S_{n}(x)$ satisfying the conditions (5) and (8), and call it the logarithmic $q$-spline of degree $n$. Unexpectedly, its representation (7) can be explicitly described (Theorem 1). In $\S 2$ we show that the global behavior of $S_{n}(x)$ is much like that of the function $\log x / \log q: S_{n}^{\prime}(x)$ is multiply monotone (Theorem 2). 
This gives a direct construction of $S_{n}(x)$ by successive integrations of the explicitly described step-function $S_{n}^{(n)}(x)$ (Corollary 1 of $\S 2$ ).

These results led us naturally to expect that the logarithmic $q$-spline $S_{n}(x)$ will converge to $\log x / \log q$ as $n \rightarrow \infty$. This, however, is not the case and the $\S \S 4,5,6,7$ describe the peculiar behavior of $S_{n}(x)$ as $n \rightarrow \infty$. Our main results are as follows. We define

$$
F(x, \theta)=\sum_{s=-\infty}^{\infty}\left(e^{-q^{\theta+s}}-e^{-x q \theta+s}\right), \quad(x>0) .
$$

This is a periodic function of $\theta$ of period 1, whose Fourier expansion (5.2) is described in $\S 5$. In terms of $F(x, \theta)$ we find that

$$
S_{n}(x)=F\left(x, \frac{\log n}{\log q}\right)+o(1) \text { as } n \rightarrow \infty,
$$

where the error term o(1) is uniform in $x$ for $x>0$ (Theorem 4 of $\S 4)$. The average value of $F(x, \theta)$ over a period in $\theta$ is $a_{0}(x)=$ $\log x / \log q$, while its total variation in a period is of the order of

$$
\left.\left|\Gamma\left(-\frac{2 \pi i}{\log q}\right)\right|=\frac{\log q}{e^{2 \pi^{2} / \log q}-e^{-2 \pi^{2} / \log q}}\right)^{1 / 2}
$$

which is small if $q$ is not too large (for $q=2$ the quantity (12) is of the order of $\left.10^{-6}\right)$. Figure 1 of Section 6 shows graphically all the terms of the relation (11) for $q=2, x=\sqrt{2}$, and $n=16,17, \cdots, 64$.

In the last section 7 it is shown that the logarithmic means of the sequence $S_{n}(x)$ do converge to the "correct" limit $\log x / \log q$ (Theorem 5). In a way this paper may be regarded as a contribution to the study of the functional equation $f(q x)=f(x)+1$.

1. Construction of the cardinal $q$-spline $S_{n}(x)$ satisfying (5) and (8). The representation (7) of our solution is explicitly described by the following

THEOREM 1 There is a unique $S_{n}(x)$ satisfying the conditions (5) and (8) and it is given by

$$
\begin{aligned}
S_{n}(x)= & P_{n}(x)+(-1)^{n} \sum_{\nu=1}^{\infty} q^{-n \nu}\left(x-q^{\nu}\right)_{+}^{n}-\sum_{\nu=0}^{\infty} q^{n \nu}\left(q^{-\nu}-x\right)_{+}^{n}, \\
& (0<x<\infty),
\end{aligned}
$$

where

$$
P_{n}(x)=\left(\frac{n}{1}\right) \frac{x-1}{q-1}-\left(\frac{n}{2}\right) \frac{x^{2}-1}{q^{2}-1}+\cdots+(-1)^{n-1} \frac{x^{n}-1}{q^{n}-1} .
$$

We call $S_{n}(x)$ the logarithmic $q$-spline of degree $n$. 
Proof. We begin by determining the polynomial $P_{n}(x)=P(x)$ representing $S_{n}(x)=S(x)$ in the interval $(1, q)$. From the conditions (2) and (8) we obtain by differentiation

$$
q^{\nu} S^{(\nu)}(q x)=S^{(\nu)}(x), \quad(\nu=1, \cdots, n-1),
$$

and setting $x=1$ we obtain

$$
q^{\nu} P^{(\nu)}(q)-P^{(\nu)}(1)=0, \quad(\nu=1, \cdots, n-1) .
$$

Clearly $P(1)=0, P(q)=1$, and therefore

$$
P(q)-P(1)=1 \text {. }
$$

We may summarize the relations (1.4) and (1.5) by saying that the polynomial $P(q x)-P(x)$ assumes for $x=1$ the value 1 to the $n$th order. This means that for an appropriate constant $c$ we have the identity

$$
P(q x)-P(x)=1+c(x-1)^{n} .
$$

The left side vanishing if $x=0$, we must have $c=(-1)^{n-1}$ and (1.6) becomes

$$
P(q x)-P(x)=1-(1-x)^{n} .
$$

All $P(x) \in \pi_{n}$ satisfying (1.7) are easily determined. Writing

$$
P(x)=a_{0}+a_{1} x+\cdots+a_{n} x^{n},
$$

substituting into (1.7) and comparing coefficients on both sides, we obtain that

$$
a_{1}(q-1)=\left(\begin{array}{l}
n \\
1
\end{array}\right), a_{2}\left(q^{2}-1\right)=-\left(\begin{array}{l}
n \\
2
\end{array}\right), \cdots, a_{n}\left(q^{n}-1\right)=(-1)^{n-1} .
$$

Therefore all solutions of (1.7) are of the form.

$$
P(x)=a_{0}+\left(\begin{array}{l}
n \\
1
\end{array}\right) \frac{x}{q-1}-\left(\begin{array}{l}
n \\
2
\end{array}\right) \frac{x^{2}}{q^{2}-1}+\cdots+(-1)^{n-1} \frac{x^{n}}{q^{n}-1} .
$$

Finally, the condition $P(1)=0$ shows that $P_{n}(x)$ indeed has the form (1.2). We may retrace our steps and see that the polynomial (1.2) satisfies (1.7), hence (1.4) and that $P(1)=0, P(q)=1$.

The polynomial $P_{n}(x)$ representing $S_{n}(x)$ in the interval $(1, q)$ having been determined, we could now extend the definition of $S_{n}(x)$ to all of $(0, \infty)$ by the representation (7) and the procedure described for determining the coefficients $a_{\nu}$. However, it is better to proceed as follows: We extend the definition of $S_{n}(x)$ from the interval $[1, q]$, where $S_{n}(x)=P_{n}(x)$, to all positive values of $x$ by means of 
the functional equation

$$
S_{n}(q x)=S_{n}(x)+1 .
$$

However, now we must verify that the condition $S(x) \in C^{n-1}(0, \infty)$ is satisfied. By iteration of (1.9), i.e. replacing $x$ by $q x, q^{2} x, \cdots$ and adding the results, we find that

$$
\begin{array}{ll}
S(x)=P\left(\frac{x}{q^{r-1}}\right)+r-1 & \text { if } q^{r-1}<x<q^{r}, \\
S(x)=P\left(\frac{x}{q^{r}}\right)+r & \text { if } q^{r}<x<q^{r+1} .
\end{array}
$$

From (1.11) and letting $x$ decrease to $q^{r}$ we obtain that $S\left(q^{r}+0\right)=$ $P(1)+r=r$, while (1.10) on letting $x$ increase to $q^{r}$ we find $S\left(q^{r}-\right.$ $0)=P(q)+r-1=1+r-1=r$. Therefore $S\left(q^{r}+0\right)=S\left(q^{r}-0\right)$ and $S(x) \in C(0, \infty)$. There remains to show that

$$
S^{(\nu)}\left(q^{r}+0\right)=S^{(\nu)}\left(q^{r}-0\right), \quad(\nu=1, \cdots, n-1) .
$$

However, on differentiating (1.10) and (1.11), we obtain

$$
S^{(\nu)}\left(q^{r}-0\right)=\frac{1}{q^{\nu(r-1)}} P^{(\nu)}(q) \text { and } S^{(\nu)}\left(q^{r}+0\right)=\frac{1}{q^{\nu r}} P^{(\nu)}(1),
$$

respectively. Now the relations (1.12) amount to $q^{\nu} P^{(\nu)}(q)=P^{(\nu)}(1)$, and these are precisely the boundary conditions (1.4) satisfied by our polynomial (1.2).

We are still to determine the explicit values of the coefficient $a_{\nu}$ of (7), as described by (1.1). If $x \neq q^{r}$, for all integers $r$, then we may differentiate the relation (1.9) even $n$ times when (1.13) becomes, in view of (1.2),

$$
\begin{aligned}
& S^{(n)}\left(q^{r}-0\right)=\frac{1}{q^{n(r-1)}} P^{(n)}(q)=\frac{q^{n}}{q^{n r}}(-1)^{n-1} \frac{n !}{q^{n}-1}, \\
& S^{(n)}\left(q^{r}+0\right)=\frac{1}{q^{n r}} P^{(n)}(1)=\frac{1}{q^{n r}}(-1)^{n-1} \frac{n !}{q^{n}-1} .
\end{aligned}
$$

These values show that the coefficients $a_{r}$ of (7) are equal to

$$
a_{r}=\frac{1}{n !}\left\{S^{(n)}\left(q^{r}+0\right)-S^{(n)}\left(q^{r}-0\right)\right\}=\frac{1}{n ! q^{n r}}(-1)^{n} n ! \text { if } r \geqq 1,
$$

and

$$
\begin{array}{r}
a_{r}=(-1)^{n-1} \frac{1}{n !}\left\{S^{(n)}\left(q^{r}+0\right)-S^{(n)}\left(q^{r}-0\right)\right\}=\frac{(-1)^{n-1}}{n ! q^{n r}}(-1)^{n} n ! \\
\text { if } r \leqq 0 .
\end{array}
$$


Therefore

$$
a_{r}= \begin{cases}(-1)^{n} q^{-n r} & \text { if } r \geqq 1, \\ -q^{-n r} & \text { if } r \leqq 0 .\end{cases}
$$

This establishes (1.1) and also Theorem 1.

2. The nice global behavior of $S_{n}(x)$. The function

$$
f(x)=\frac{\log x}{\log q}
$$

that we are interpolating by $S_{n}(x)$ has the derivative $(x \log q)^{-1}$ which is completely monotone in $(0, \infty)$, because

$$
f^{(\nu)}(x)=\frac{(-1)^{\nu-1}}{\log q} \frac{(\nu-1) !}{x^{\nu}}, \quad(\nu=1,2, \cdots) .
$$

To what extent does $S_{n}(x)$ imitate this regular behavior of $f(x)$ ? That it does so as best as it can (remember that $S_{n}(x)$ has only $n$ derivatives, the $n$th being a step-function) is shown by

THEOREM 2. The logarithmic $q$-spline $S_{n}(x)$ has the properties

$$
\begin{gathered}
(-1)^{\nu-1} S_{n}^{(\nu)}(x)>0 \text { in } 0<x<\infty, \\
S_{n}^{(\nu)}(x)=O\left(x^{-\nu}\right) \text { for } \nu=1,2, \cdots, n .
\end{gathered}
$$

Proof. We first establish (2.4). Differentiation of (1.9) gives $q^{\nu} S^{(\nu)}(q x)=S^{(\nu)}(x)$, where $x \neq q^{r}$ for all $r$, if $\nu=n$. Iterating this we obtain that

$$
S^{(\nu)}\left(q^{r} x\right)=q^{-\nu r} S^{(\nu)}(x) \quad(r \text { integer }) .
$$

Let us write

$$
M_{\nu}=\sup _{1<x<q}\left|S^{(\nu)}(x)\right| .
$$

Assuming that

$$
q^{r}<x<q^{r+1},
$$

then (2.5) and (2.6) show, on replacing $x$ by $x q^{-r}$, that

$$
\left|S^{(\nu)}(x)\right| \leqq M_{\nu} q^{-\nu r}=M q^{-\nu(r+1)} q^{\nu}<M_{\nu} x^{-\nu} q^{\nu}
$$

and therefore

$$
\left|S^{(\nu)}(x)\right|<\left(M_{\nu} q^{\nu}\right) x^{-\nu}
$$


As the right side does not depend on $r$, the condition (2.7) becomes irrelevant and (2.8) holds for all positive $x$. This establishes (2.4).

We now establish (2.3) by induction for decreasing values of $\nu$. (2.3) is true for $\nu=n$ : This is shown by (1.15) which gives

$$
S_{n}^{(n)}(x)=(-1)^{n-1} \frac{n !}{q^{n}-1}\left(q^{r}\right)^{-n} \text { if } q^{r}<x<q^{r+1} .
$$

Therefore (2.3) holds for $\nu=n$. Assuming that (2.3) holds for a value of $\nu(\nu>1)$, let us establish $(2.3)$ for $\nu-1$ in place of $\nu$. By (2.4) we can write

$$
S_{n}^{(\nu-1)}(x)=-\int_{x}^{\infty} S_{n}^{(\nu)}(t) d t \quad(x>0),
$$

and this already shows that $S_{n}^{(\nu-1)}(x)$ has the correct sign. This completes a proof of Theorem 2 .

We may summarize our findings by stating

COROLLARY 1. We may think of the logarithmic q-spline $S_{n}(x)$ as generated as follows: We define $S_{n}^{(n)}(x)$ as a step-function on $(0, \infty)$ by the relations (2.9), for all integral values of $r$. We integrate successively $S_{n}^{(n)}(x)$ by means of the relations (2.10) for $\nu=n, n-1$, $\cdots, 3,2$, obtaining finally the decreasing positive function $S_{n}^{\prime}(x)$. Finally, we define $S_{n}(x)$ by

$$
S_{n}(x)=\int_{1}^{x} S_{n}^{\prime}(t) d t, \quad(x>0) .
$$

That the resulting function $S_{n}(x)$ is an element of $\sum_{n, q}$, i.e. a cardinal $q$-spline, is clear because we integrate a step-function $S_{n}^{(n)}(x)$ $n$ times. What does seem remarkable in that the resulting function should solve the interpolation problem

$$
S_{n}\left(q^{\nu}\right)=\nu, \text { for all } \nu,
$$

and, what is more, even satisfy the functional equation

$$
S_{n}(q x)=S_{n}(x)+1 .
$$

3. Direct reconstruction of $S_{n}(x)$ by Corollary 1. Actually the reconstruction of $S_{n}(x)$ as described by Corollary 1 is easily carried out explicitly yielding our previous results. We start from (2.9) and abserve that this step function may certainly by written in the form

$$
S_{n}^{(n)}(x)=\sum_{s=-\infty}^{\infty} c_{s}\left(q^{s}-x\right)_{+}^{0}, \quad(x>0) .
$$


If $q^{r}<x<q^{r+1}$ then (3.1) becomes $S_{n}^{(n)}(x)=\sum_{s-r} c_{s}$ and this must equal the right side of (2.9). By differencing we obtain

$$
c_{r}=(-1)^{n-1} \frac{n !}{q^{n}-1} q^{-r n}\left(q^{n}-1\right)=(-1)^{n-1} n ! q^{-r n},
$$

and substituting into (3.1) we obtain that

$$
S_{n}^{(n)}(x)=(-1)^{n-1} n ! \sum_{r=-\infty}^{\infty} q^{-r n}\left(q^{r}-x\right)_{+}^{0}
$$

On performing $\nu$ times the operation (2.10) we obtain

$$
S_{n}^{(n-\nu)}(x)=(-1)^{\nu}(-1)^{n-1} n ! \sum_{n} q^{-r n}\left(q^{r}-x\right)_{+}^{\nu} / \nu !
$$

and in particular that

$$
S_{n}^{\prime}(x)=\sum_{-\infty}^{\infty} q^{-r} n\left(1-x q^{-r}\right)_{+}^{n-1} .
$$

The last integration (2.11) yields

$$
S_{n}(x)=\sum_{-\infty}^{\infty}\left\{\left(1-q^{-r}\right)_{+}^{n}-\left(1-x y^{-r}\right)_{+}^{n}\right\}, \quad(x>0) .
$$

If we assume that $1<x<q$, then (3.4) reduces to

$$
\begin{aligned}
S_{n}(x)= & \sum_{r=1}^{\infty}\left\{\left(1-q^{-r}\right)^{n}-\left(1-x q^{-r}\right)^{n}\right\} \\
= & \sum_{r=1}^{\infty}\left\{\left(1-\left(\begin{array}{c}
n \\
1
\end{array}\right) q^{-r}+\cdots+(-1)^{n} q^{-n r}\right)\right. \\
& \left.-\left(1-\left(\begin{array}{c}
n \\
1
\end{array}\right) x q^{-r}+\cdots+(-1)^{n} x^{n} q^{-r n}\right)\right\} .
\end{aligned}
$$

If we cancel within the brackets the two unit terms, then the resulting series may be summed termwise and we obtain that

$$
\begin{aligned}
S_{n}(x)= & \left(\begin{array}{l}
n \\
1
\end{array}\right)(x-1) \frac{q^{-1}}{1-q^{-1}}-\left(\begin{array}{l}
n \\
2
\end{array}\right)\left(x^{2}-1\right) \frac{q^{-2}}{1-q^{-2}} \\
& +\cdots+(-1)^{n-1}\left(x^{n}-1\right) \frac{q^{-n}}{1-q^{-n}} \\
= & \left(\begin{array}{l}
n \\
1
\end{array}\right) \frac{x-1}{q-1}-\left(\begin{array}{l}
n \\
2
\end{array}\right) \frac{x^{2}-1}{q^{2}-1}+\cdots+(-)^{n-1} \frac{x^{n}-1}{q^{n}-1} \\
& \text { in } 1<x<q,
\end{aligned}
$$

which agrees with the result of Theorem 1. The expression (3.4) will be found useful in our next section. 
4. The peculiar behavior of $S_{n}(x)$ as $n \rightarrow \infty$. We have derived in (3.4) the expression

$$
S_{n}(x)=\sum_{-\infty}^{\infty}\left\{\left(1-q^{-r}\right)_{+}^{n}-\left(1-x q^{-r}\right)_{+}^{n}\right\}, \quad(0<x<\infty) .
$$

This expression will show against all expectations that the $q$-spline $S_{n}(x)$ does not converge to $f(x)=\log x / \log q$ as $n \rightarrow \infty$. The key to our discussion is the following way of writing the series (4.1) Using the square brackets to denote the integral part we define

$$
\theta_{n}=\frac{\log n}{\log q}-\left[\frac{\log n}{\log q}\right]=\frac{\log n}{\log q}-k_{n},
$$

hence $n=q^{\theta_{n}+k_{n}}$ and therefore $1=q^{\theta_{n}+k_{n}} / n$. We may therefore rewrite (4.1) as

$$
S_{n}(x)=\sum_{r}\left\{\left(1-\frac{1}{n} q^{\theta_{n}+k_{n}-r}\right)_{+}^{n}-\left(1-\frac{1}{n} x q^{\theta_{n}+k_{n}-r}\right)_{+}^{n}\right\}
$$

and finally as

$$
S_{n}(x)=\sum_{s=-\infty}^{\infty}\left\{\left(1-\frac{1}{n} q^{\theta_{n}+s}\right)_{+}^{n}-\left(1-\frac{1}{n} x q^{\theta_{n}+s}\right)_{+}^{n}\right\} .
$$

We also define the function

$$
F(x, \theta)=\sum_{s=-\infty}^{\infty}\left\{\left(e^{-q^{\theta+s}}-e^{-x q^{\theta+s}}\right), \quad(x>0,-\infty<\theta<\infty)\right.
$$

which is evidently periodic in $\theta$ of period 1 . For each fixed positive $x$ the function $F(x, \theta)$ of $\theta$ has an interval of variability

$$
I(x)=\left[\min _{\theta} F(x, \theta), \max _{\theta} F(x, \theta)\right]
$$

in terms of which we can state the following

THEOREM 3. For each fixed positive $x$ the set of limit points of the sequence $\left(S_{n}(x)\right)_{n=1,2, \ldots}$ is identical with the interval $I(x)$ defined by (4.5).

Our discussion will simplify if we consider the derivative with respect to $x$ of the functions (4.3) and (4.4), hence

$$
S_{n}^{\prime}(x)=\sum_{s} q^{\theta_{n}+s}\left(1-\frac{1}{n} x q^{\theta_{n}+s}\right)_{+}^{n-1}
$$

and

$$
F_{x}(x, \theta)=\sum_{s} q^{\theta+s} e^{-x q^{\theta+s}}
$$


At this point we need some lemmas.

LEMMA 1. If $a_{n}$ and $a$ are real, $a_{n} \rightarrow a$, then

$$
\lim _{n \rightarrow \infty}\left(1-\frac{a_{n}}{n}\right)_{+}^{n-1}=e^{-a} \text {. }
$$

We may omit the elementary proof.

LEMMa 2. The inequality

$$
\left(1-\frac{x}{n}\right)^{n-1}<2 e^{-x} \text { holds for } x \geqq 0, n \geqq 2 \text {. }
$$

Proof. (4.9) is trivial if $x>n$. If $0 \leqq x \leqq n$ we consider

$$
g(x)=\left(1-\frac{x}{n}\right)^{n-1} e^{x}
$$

and observe that

$$
\begin{aligned}
g^{\prime}(x) & =\left(1-\frac{x}{n}\right)^{n-1} e^{x}-\frac{n-1}{n}\left(1-\frac{x}{n}\right)^{n-2} e^{x} \\
& =e^{x}\left(1-\frac{x}{n}\right)^{n-2}\left\{1-\frac{x}{n}-\frac{n-1}{n}\right\} \\
& =\frac{1}{n} e^{x}\left(1-\frac{x}{n}\right)^{n-2}(1-x) .
\end{aligned}
$$

This shows that in $[0, n]$ we have

$$
\max g(x)=g(1)=\left(1-\frac{1}{n}\right)^{n-1} \cdot e \rightarrow e^{-1} \cdot e=1 \text { as } n \rightarrow \infty .
$$

Actually $\left(1-n^{-1}\right)^{n-1} e<2$ if $n \geqq 2$ which proves the lemma.

LEMma 3. The function $F(x, \theta)$, defined by (4.4), satisfies the functional equation

$$
F(q x, \theta)=F(x, \theta)+1, \quad(x>0) .
$$

Proof. By (4.4)

$$
\begin{aligned}
F(q x, \theta) & =\sum_{s}\left(e^{-q^{\theta+s}}-e^{-x q \theta+s+1}\right) \\
& =\sum_{s}\left(e^{-q^{\theta+s}}-e^{-x q^{\theta+s}}+e^{-x q^{\theta+s}}-e^{x q^{\theta+s+1}}\right),
\end{aligned}
$$

hence 


$$
F(q x, \theta)=F(x, \theta)+\sum_{s}\left(e^{-x q^{\theta+s}}-e^{-x q^{\theta+s+1}}\right) .
$$

Writing $x q^{\theta}=\alpha$, the last series becomes $\sum_{s}\left(e^{-\alpha q^{s}}-e^{-\alpha q^{s+1}}\right)$ and this is easily shown to converge to the sum 1 as follows: Letting $A \rightarrow$ $-\infty$ and $B \rightarrow+\infty$ we have

$$
\begin{aligned}
\sum_{s}\left(e^{-\alpha q^{s}}-e^{-\alpha q^{s+1}}\right) & =\lim \sum_{s=A}^{B-1}\left(e^{-\alpha q^{s}}-e^{-a q^{s+1}}\right) \\
& =\lim \left(e^{-\alpha q^{A}}-e^{-\alpha q^{B}}\right)=1-0=1 .
\end{aligned}
$$

Observe that the functional equations (8) and (4.10) are identical. It follows that the relation

$$
S_{n}(q x)-F(q x, \theta)=S_{n}(x)-F(x, \theta)
$$

holds for all positive $x$ and all real $\theta$. Of special interest for us is the following

COROLLARY 2. In studying the behavior of the difference $S_{n}(x)-$ $F\left(x, \theta_{n}\right)$, we loose no generality in assuming that $x$ is restricted to the interval $1 \leqq x \leqq q$.

\section{LEMMA 4. Let}

(4.12) $2 \leqq n_{1}<n_{2}<\cdots$ be an increasing sequence of integers,

$$
\eta_{1}, \eta_{2}, \cdots \text { be a sequence of reals, } 0 \leqq \eta_{2} \leqq 1 \text {, }
$$

and let $x$ be restricted to the interval

$$
1 \leqq x \leqq q
$$

Then the series

$$
\sum_{s=-\infty}^{\infty} q^{\eta_{\nu}+s}\left(1-x \frac{q^{\eta_{\nu}+s}}{n_{\nu}}\right)_{+}^{n_{\nu}-1}
$$

converges uniformly with respect to all data $n_{\nu}, \eta_{\nu}$ and $x$ satisfying the conditions (4.12), (4.13) and (4.14).

Proof. By Lemma 2 the series (4.15) is termwise dominated by the series

$$
\sum_{s} q^{\eta+s} 2 e^{-x q_{\nu}+s}
$$

and this last series is termwise dominated by the series

$$
\sum_{s=-\infty}^{\infty} q^{1+s} 2 e^{-q^{s}}
$$


whose terms are independent of all the data. Since (4.16) converges, the lemma is established.

LEMMA 5. If we add to (4.13) the assumption

$$
\lim _{\nu \rightarrow \infty} \eta_{\nu}=\eta
$$

then

$$
\lim _{\nu \rightarrow \infty} \sum_{s} q^{\eta_{\nu}+s}\left(1-x \frac{q^{\eta_{\nu}+s}}{n_{\nu}}\right)_{+}^{n_{\nu}-1}=\sum_{s} q^{\eta+s} e^{-x q^{\eta+s}}
$$

uniformly in $x$ satisfying (4.14).

Proof. By Lemma 1

$$
\lim _{\nu \rightarrow \infty} q^{\eta_{\nu}+s}\left(1-x \frac{q^{\eta_{\nu}+s}}{n_{\nu}}\right)_{+}^{n_{\nu}+1}=q^{\eta+s} e^{-x q \eta+s},
$$

and the uniform convergence of the series on the left side of (4.18) (Lemma 4), implies the relation (4.18).

In view of the uniform convergence in $x$ we can integrate termwise the limit relation (4.18) with respect to $x$ between the limits of integration 1 and $x$, where we assume (4.14). These integrations are immediately performed because the expressions to be integrated were obtained by (4.6) and (4.7) by differentiation. We state the result as

LEMma 6. If we assume (4.12), (4.13), (4.14), and (4.17) to hold, then

$$
\begin{gathered}
\lim _{\nu \rightarrow \infty} \sum_{s}\left\{\left(1-\frac{1}{n_{\nu}} q^{\eta_{\nu}+s}\right)_{+}^{n_{\nu}}-\left(1-\frac{x}{n_{\nu}} q^{\eta_{\nu}+s}\right)_{+}^{n_{\nu}}\right\} \\
=\sum_{s}\left(e^{-q \eta+s}-e^{-x q \eta+s}\right),
\end{gathered}
$$

uniformly in $\eta_{\nu}, \eta$, and in $x$.

An approximation of the $q$-spline $S_{n}(x)$ is now obtained as follows. We apply Lemma 6 for the special case that $n_{\nu}=\nu$, and that $\eta_{\nu}$ does not depend on $\nu, \eta_{\nu}=\eta$ for all $\nu$. We obtain from (4.19) that

(4.20) $\lim _{n \rightarrow \infty} \sum_{s}\left\{\left(1-\frac{1}{n} q^{\eta+s}\right)_{+}^{n}-\left(1-\frac{x}{n} q^{\eta+s}\right)_{+}^{n}\right\}=\sum_{s}\left(e^{-q^{\eta+s}}-e^{-x q^{\eta+8}}\right)$, uniformly in $\eta(0 \leqq \eta \leqq 1)$ and in $x(1 \leqq x \leqq q)$.

This means the following: To every positive $\varepsilon$, there corresponds 
an $N_{\varepsilon}$ such that the error term in (4.20) is in absolute value $<\varepsilon$ if $n>N_{\varepsilon}$, and this for all $\eta$ and $x$ in their respective intervals. But then it is clear that we may let $\eta$ also depend on $n$. Choosing in particular

$$
\eta=\theta_{n}=\log n / \log q-[\log n / \log q]
$$

we obtain by (4.3) and (4.4) the following

THEOREM 4. We have the relation

$$
S_{n}(x)=F\left(x, \frac{\log n}{\log q}\right)+o(1) \text { as } n \rightarrow \infty,
$$

the error term o(1) being uniform for all positive values of $x$.

Notice that indeed $F(x, \log n / \log q)=F\left(x, \theta_{n}\right)$, due to the periodicity of $F(x, \theta)$ as a function of $\theta$. In extending the uniformity of $o(1)$ from $[1, q]$ to $(0, \infty)$ we have used Corollary 2 .

The numbers $\theta_{n}$ being $\equiv \log n / \log q, \bmod 1$, it is clear that the sequence $\left(\theta_{n}\right)$ is everywhere dense in $[0,1]$. Given $\theta$ in $[0,1]$ we can therefore choose an increasing sequence $\left(n_{\nu}\right)$ such that $\theta_{n_{\nu}} \rightarrow \theta$ as $\nu \rightarrow \infty$. It now follows from (4.21) and the continuity of $F(x, \theta)$ that

$$
\lim _{\nu \rightarrow \infty} S_{n_{\nu}}(x)=F(x, \theta) .
$$

In view of these remarks it is clear that the relation (4.21) implies the truth of Theorem 3 .

5. The Fourier series of $F(x, \theta)$. Surely Theorem 4 shows the role played by the periodic function

$$
F(x, \theta)=\sum_{s=-\infty}^{\infty}\left(e^{-q^{\theta+s}}-e^{-x q^{\theta+s}}\right)
$$

in the behavior of $S_{n}(x)$ for large values of $n$. Let us derive its Fourier expansion

$$
F(x, \theta)=\sum_{\nu=-\infty}^{\infty} a_{\nu}(x) e^{2 \pi i \nu \theta}
$$

We find that

$$
a_{0}(x)=\int_{0}^{1} \sum_{s}\left(e^{-q^{\theta+s}}-e^{-x q^{\theta+s}}\right) d \theta=\int_{-\infty}^{\infty}\left(e^{-q^{t}}-e^{-x q^{t}}\right) d t
$$

and setting $q^{t}=u$, hence $q^{t} \log q d t=d u$, we find that 


$$
\begin{aligned}
a_{0}(x) & =\frac{1}{\log q} \int_{0}^{\infty}\left(e^{-u}-e^{-x u}\right) u^{-1} d u=\frac{1}{\log q} \int_{0}^{\infty}\left(\int_{1}^{x} e^{-u v} d v\right) d u \\
& =\frac{1}{\log q} \int_{1}^{x}\left(\int_{0}^{\infty} e^{-u v} d u\right) d v=\frac{1}{\log q} \int_{1}^{x} \frac{d v}{v}
\end{aligned}
$$

and finally

$$
a_{0}(x)=\frac{\log x}{\log q}
$$

Notice that the average of $F(x, \theta)$ over a period is equal to the presumed limit of $S_{n}(x)$. At this point we observe that $F(x, \theta)$ being continuous in $\theta$, there certainly exists $a \theta_{x}$ such that

$$
F\left(x, \theta_{x}\right)=\log x / \log q \text {. }
$$

The last paragraph of $\S 4$ now implies the

CoRollary 2. To every positive $x$ corresponds a sequence $\left(n_{\nu}\right)$ such that

$$
\lim _{\nu \rightarrow \infty} S_{n_{\nu}}(x)=\frac{\log x}{\log q} .
$$

If $\nu \neq 0$, we find that

$$
\begin{aligned}
a_{\nu}(x) & =\int_{0}^{1} e^{-2 \pi i \nu \theta} F(x, \theta) d \theta=\int_{0}^{1} e^{-2 \pi i \nu \theta} \sum_{s}\left(e^{-q^{\theta+s}}-e^{-x q^{\theta+s}}\right) d \theta \\
& =\sum_{s} \int_{0}^{1} e^{-2 \pi i v \theta}\left(e^{-q \theta+s}-e^{-x q \theta+s}\right) d \theta \\
& =\int_{-\infty}^{\infty} e^{-2 \pi i \nu t}\left(e^{-q^{t}}-e^{-x q^{t}}\right) d t .
\end{aligned}
$$

Setting again $q^{t}=u$ we obtain

$$
a_{\nu}(x)=\frac{1}{\log q} \int_{0}^{\infty} u^{-(2 \pi i \nu / \log q)-1}\left(e^{-u}-e^{-x u}\right) d u .
$$

This is easily evaluated in terms of the $\Gamma$-function. If we add $\varepsilon(\varepsilon>0)$ to the exponent of $u$ in the integrand, we may then write the integral as a difference of two $\Gamma$-integrals which amounts to

$$
\frac{1}{\log q} \Gamma\left(\varepsilon-\frac{2 \pi i \nu}{\log q}\right)\left(1-x^{(2 \pi i \nu / \log q)-\varepsilon}\right) \text {. }
$$

On letting $\varepsilon \rightarrow 0$ we obtain that

$$
a_{\nu}(x)=\frac{1}{\log q} \Gamma\left(-\frac{2 \pi i \nu}{\log q}\right)\left(1-x^{2 \pi i \nu / \log q}\right), \quad(\nu \neq 0) .
$$


From (4.4) we see that $F(1, \theta)=0$ for all $\theta$. Moreover (4.10) shows that $F\left(q^{r}, \theta\right)=r$ for all $\theta$. We therefore see that the interval of variability $I(x)$, defined by (4.5), reduces of the point $r$ if $x=q^{r}$ ( $r$ integer). The relations (5.6) allow us to prove the converse, to the effect that $I(x)$ reduces to a single point, namely $\log x / \log q$, only if $x$ is a power of $q$. For if $I(x)$ is a single point, then the unicity theorem of Fourier series shows that all coefficients (5.6) vanish. The $\Gamma$-function being $\neq 0$, we conclude that

$$
1=x^{2 \pi i \nu / \log q}=e^{2 \pi i \nu(\log x / \log q)} \text { if } \nu \neq 0
$$

and this indeed implies that $\log x / \log q=r$ is an integer, hence $x=q^{r}$.

In view of Theorem 3 we have just established

COROLlary 3. The relation

$$
\lim _{n \rightarrow \infty} S_{n}(x)=\frac{\log x}{\log q}
$$

is valid if and only if $x=q^{r}$ for an integer $r$.

The Fourier series (5.2), (5.3), (5.6) allows us to write the approximation formula (4.21) in explicit terms. Observing that

$$
e^{2 \pi i \nu(\log n / \log q)}=n^{2 \pi i \nu / \log q}
$$

and using the Fourier series we obtain the following

CoRollary 4. For every fixed positive $x$ we have

$$
\begin{aligned}
S_{n}(x)= & \frac{\log x}{\log q}+\frac{1}{\log q} \sum_{\nu \neq 0} \Gamma\left(-\frac{2 \pi i \nu}{\log q}\right)\left(1-x^{2 \pi i \nu / \log q}\right) n^{2 \pi i \nu / \log q} \\
& +o(1), \text { as } n \rightarrow \infty,
\end{aligned}
$$

the error term o(1) being uniform in $x>0$.

This will be used in $\S 7$.

6. A graph for the case $q=2$ and $x=\sqrt{2}$. We select

$$
q=2, x=\sqrt{2} \text {, hence } \frac{\log x}{\log q}=\frac{1}{2} .
$$

Figure 1 shows the graph of the function

$$
y=F(\sqrt{2}, \theta) \text { in the interval } 4 \leqq \theta \leqq 6
$$

and also the 49 points 


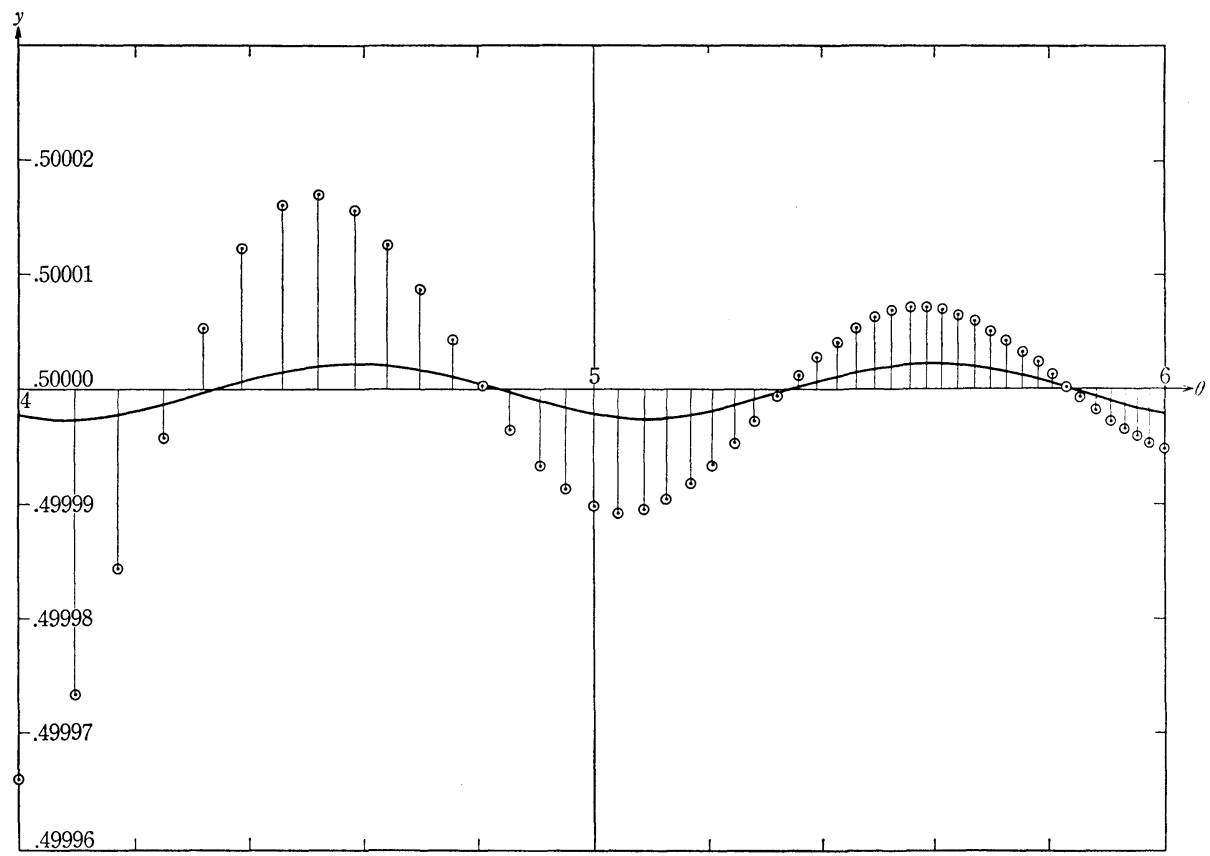

This sheet represents in the $(\theta, y)$-plane the rectangle defined by

$$
4 \leqq \theta \leqq 6, \quad .49996 \leqq y \leqq .50003 .
$$

The curve is $y=F(\sqrt{2}, \theta), 4 \leqq \theta \leqq 6$.

The 49 dots represent the points $\left(\log n / \log 2, S_{n}(\sqrt{2})\right), n=16,17, \cdots, 64$.

Figure 1

$$
\left(\frac{\log n}{\log 2}, S_{n}(\sqrt{2})\right) \text { for } n=16,17, \cdots, 64
$$

Notice the tremendously magnified scale in the $y$-direction, and that the abscissae of the points (6.3) also vary from 4 to 6 . Thus Figure 1 exhibits graphically all terms of the relation (4.21) or

$$
S_{n}(\sqrt{2})=F\left(\sqrt{2}, \frac{\log n}{\log 2}\right)+o(1) \text { for } n=16,17, \cdots, 64 .
$$

If we were to extend the graph of Figure 1 from $4 \leqq \theta \leqq 6$ to $4 \leqq$ $\theta \leqq+\infty$, then the abscissae of consecutive dots would get closer together indefinitely, while the oscillations of their ordinates would decrease so that the dots would tend to the curve $y=F(\sqrt{2}, \theta)$, according to the relation (6.4).

Figure 1 reveals the following apparent situation: The "curve" through the points (6.3) seems to intersect the horizontal line $y=1 / 2$ in practically the same points as the curve $y=F(\sqrt{2}, \theta)$. It therefore seems that $S_{n}(\sqrt{2})$ is very close to its "correct" limit $1 / 2$ whenever $n$ is such that $\log n / \log 2$ is close to one of the points $\theta$ 
where $F(\sqrt{2}, \theta)$ assumes its average value $1 / 2$. We have no explanation for this phenomenon, if true.

A word on the computations underlying Figure 1. In the first place we needed the ordinates of the points (6.3). Since $x=\sqrt{2}$ is in the interval $[1, q]$ we have $S_{n}(x)=P_{n}(x)$, by Theorem 1. Moreover the relation (1.2) shows that

$$
\frac{\log x}{\log q}-P_{n}(x)=\left.(-1)^{n} \Delta^{n} \frac{x^{t}-1}{q^{t}-1}\right|_{t=0} .
$$

Here $\Delta^{n}$ denotes the ordinary $n$th order forward difference (with step 1) with respect to $t$. Our colleague C. de Boor kindly computed these differences for the data (6.1) and for $n=1,2,3, \cdots, 70$. Beyond this point round-off difficulties became apparent. This gave us the points (6.3).

The function (6.2) was obtained from its Fourier series (5.2). It so happens that for $x=\sqrt{2}$ the coefficients (5.6) vanish for all even values of $\nu \neq 0$. For this reason by taking only the fundamental $(\nu= \pm 1)$ in the expansion (5.2) we already obtain better than 15-place accuracy. The coefficients $a_{1}(\sqrt{2})$ and $a_{-1}(\sqrt{2})$ were computed by means of the fine tables in [1], (p. 277), and we found with 11-place accuracy that

$$
F(\sqrt{2}, \theta)=\frac{1}{2}+A \sin 2 \pi(\theta-\alpha),
$$

where

$$
A=.238061 \times 10^{-5}, \quad \alpha=.340775 \text {. }
$$

7. The summability of the sequence $S_{n}(x)$. We rewrite (5.8) as

$$
S_{n}(x)=\frac{\log x}{\log q}+\sum_{\nu \neq 0} a_{\nu}(x) n^{2 \pi i \nu / \log q}+o(1)
$$

and are looking for a summability method that will produce the limit $\log x / \log q$. For this it is necessary that the method should assign for the sequence

$$
\left(n^{i \lambda}\right)_{n=1,2, \ldots} \quad(\text { for constant } \lambda \neq 0)
$$

the limit zero. Neither the Cesàro nor the Abel method will do that. However, the logarithmic means (see [2], $\$ \S 3.8$ and 4.16) will be found to work yielding the following 


$$
\lim _{n \rightarrow \infty} \frac{1}{\log n}\left\{S_{1}(x)+\frac{1}{2} S_{2}(x)+\cdots+\frac{1}{n} S_{n}(x)\right\}=\frac{\log x}{\log q}
$$

uniformly in $x, x>0$.

Proof. Referring to (7.1) and using the fact that

$$
\sum_{\nu \neq 0}\left|a_{\nu}(x)\right|<\frac{2}{\log q} \sum_{\nu \neq 0}\left|\Gamma\left(-\frac{2 \pi i \nu}{\log q}\right)\right|<\infty,
$$

it is clear that (7.1) will imply (7.3) as soon as we use the following

Lemma 6. For real $\lambda$ we have

$$
\left|1^{i \lambda-1}+2^{i \lambda-1}+\cdots+n^{i \lambda-1}\right| \leqq 1+\log n \text { for all } n,
$$

and

$$
\lim _{n \rightarrow \infty} \frac{1}{\log n}\left(1^{i \lambda-1}+2^{i \lambda-1}+\cdots+n^{i \lambda-1}\right)=0 \text { if } \lambda \neq 0 .
$$

Proof. 1. The left side of (7.4) is less than

$$
1+\frac{1}{2}+\cdots+\frac{1}{n} \leqq 1+\int_{1}^{n} \frac{d x}{x}=1+\log n \text {. }
$$

2. Writing $f(x)=x^{i \lambda-1}$ we use the Euler-MacLaurin summation formula

$$
\sum_{1}^{n} f(k)=\int_{1}^{n} f(x) d x+\frac{1}{2}(f(1)+f(n))+\int_{1}^{n}\left(x-[x]-\frac{1}{2}\right) f^{\prime}(x) d x
$$

which shows that

$$
\begin{aligned}
\left|\sum_{1}^{n} f(k)\right| & <\left|\int_{1}^{n} f(x) d x\right|+\frac{1}{2}\left(1+n^{-1}\right)+\int_{1}^{n}\left|f^{\prime}(x)\right| d x \\
& =\left|n^{i \lambda}-1\right| \lambda^{-1}+\frac{1}{2}\left(1+n^{-1}\right)+|i \lambda-1| \int_{1}^{n} x^{-2} d x .
\end{aligned}
$$

The last expression is $O(1)$ and therefore $o(\log n)$.

\section{REFERENCES}

1. Milton Abramowitz and Irene A. Stegun, Editors, Handbook of Mathematical Functions..., Nat. Bureau of Standards, Washington, D. C., 1965.

2. G. H. Hardy, Divergent series, Oxford 1949.

3. I. J. Schoenberg, Cardinal spline interpolation, Regional Conference Monograph No. 12, SIAM, Philadelphia, 1973.

Received May 22, 1975 . Sponsored by the United States Army under Contract No. DA-31-124-ARO-D-462.

YESHIVA UNIVERSITY 


\section{PACIFIC JOURNAL OF MATHEMATICS}

\section{EDITORS}

RICHARD ARENS (Managing Editor) University of California

Los Angeles, California 90024

\section{R. A. BEAUMONT}

University of Washington Seattle, Washington 98105
J. DugundjI Department of Mathematics University of Southern Californı Los Angeles, California 90007

D. Gilbarg AND J. Milgram Stanford University Stanford, California 94305

\section{ASSOCIATE EDITORS}

E. F. BECKENBACH

B. H. NeUMANN

F. WOLF

K. YosHidA

\section{SUPPORTING INSTITUTIONS}

UNIVERSITY OF BRITISH COLUMBIA CALIFORNIA INSTITUTE OF TECHNOLOGY UNIVERSITY OF CALIFORNIA MONTANA STATE UNIVERSITY UNIVERSITY OF NEVADA NEW MEXICO STATE UNIVERSITY OREGON STATE UNIVERSITY UNIVERSITY OF OREGON OSAKA UNIVERSITY

\author{
UNIVERSITY OF SOUTHERN CALIFORNIA \\ STANFORD UNIVERSITY \\ UNIVERSITY OF TOKYO \\ UNIVERSITY OF UTAH \\ WASHINGTON STATE UNIVERSITY \\ UNIVERSITY OF WASHINGTON \\ AMERICAN MATHEMATICAL SOCIETY \\ NAVAL WEAPONS CENTER
}




\section{Pacific Journal of Mathematics}

\section{Vol. 61, No. $1 \quad$ November, 1975}

Jiří Adámek, V. Koubek and Věra Trnková, Sums of Boolean spaces represent every

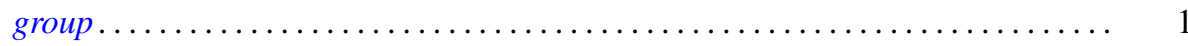

Richard Neal Ball, Full convex l-subgroups and the existence of $a^{*}$-closures of

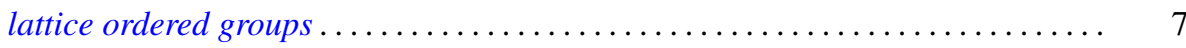

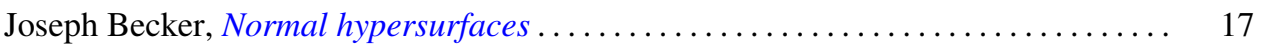

Gerald A. Beer, Starshaped sets and the Hausdorff metric . . . . . . . . . . . . . 21

Dennis Dale Berkey and Alan Cecil Lazer, Linear differential systems with

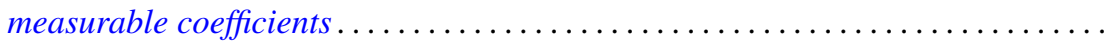

Harald Boehme, Glättungen von Abbildungen 3-dimensionaler

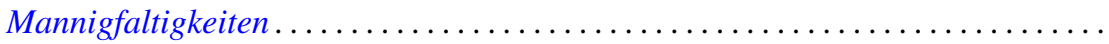

Stephen LaVern Campbell, Linear operators for which $T^{*} T$ and $T+T^{*}$

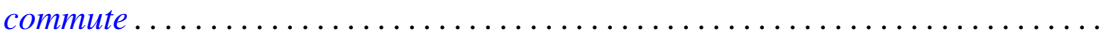

H. P. Dikshit and Arun Kumar, Absolute summability of Fourier series with

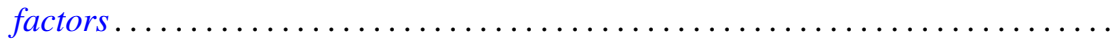

Andrew George Earnest and John Sollion Hsia, Spinor norms of local integral

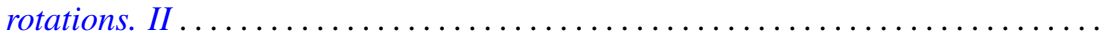

Erik Maurice Ellentuck, Semigroups, Horn sentences and isolic structures .........

Ingrid Fotino, Generalized convolution ring of arithmetic functions . . . . . . . . . . .

Michael Randy Gabel, Lower bounds on the stable range of polynomial rings .......

Fergus John Gaines, Kato-Taussky-Wielandt commutator relations and

characteristic curves

Theodore William Gamelin, The polynomial hulls of certain subsets of $C^{2}$

R. J. Gazik and Darrell Conley Kent, Coarse uniform convergence spaces. . .

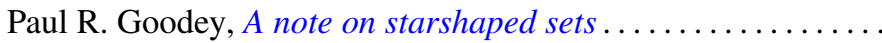

Eloise A. Hamann, On power-invariance

M. Jayachandran and M. Rajagopalan, Scattered compactification for $N \cup\{P\}$. . .

V. Karunakaran, Certain classes of regular univalent functions .

John Cronan Kieffer, A ratio limit theorem for a strongly subadditive set function in a locally compact amenable group .................

Siu Kwong Lo and Harald G. Niederreiter, Banach-Buck measure, density, and uniform distribution in rings of algebraic integers ........

Harold W. Martin, Contractibility of topological spaces onto metric spaces ....

Harold W. Martin, Local connectedness in developable spaces .

A. Meir and John W. Moon, Relations between packing and covering numbers of a tree.

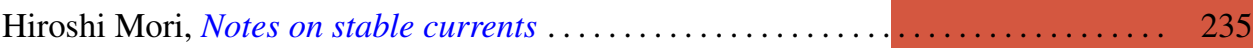

Donald J. Newman and I. J. Schoenberg, Splines and the logarithmic function . . . . 241

M. Ann Piech, Locality of the number of particles operator....

Fred Richman, The constructive theory of $K T$-modules .......

Gerard Sierksma, Carathéodory and Helly-numbers of

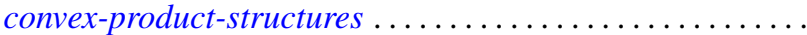

Raymond Earl Smithson, Subcontinuity for multifunctions .... . . 